\title{
Principal Component Analysis as a Tool for Analyzing Beat-to-Beat Changes in ECG Features: Application to ECG-Derived Respiration
}

\author{
Philip Langley*, Emma J. Bowers, and Alan Murray
}

\begin{abstract}
An algorithm for analyzing changes in ECG morphology based on principal component analysis (PCA) is presented and applied to the derivation of surrogate respiratory signals from single-lead ECGs. The respiratory-induced variability of ECG features, $P$ waves, $Q R S$ complexes, and $T$ waves are described by the PCA. We assessed which ECG features and which principal components yielded the best surrogate for the respiratory signal. Twenty subjects performed controlled breathing for $180 \mathrm{~s}$ at $4,6,8,10$, 12, and 14 breaths per minute and normal breathing. ECG and breathing signals were recorded. Respiration was derived from the ECG by three algorithms: the PCA-based algorithm and two established algorithms, based on RR intervals and QRS amplitudes. ECG-derived respiration was compared to the recorded breathing signal by magnitude squared coherence and cross-correlation. The top ranking algorithm for both coherence and correlation was the PCA algorithm applied to QRS complexes. Coherence and correlation were significantly larger for this algorithm than the $\mathbf{R R}$ algorithm ( $p<0.05$ and $p<0.0001$, respectively) but were not significantly different from the amplitude algorithm. PCA provides a novel algorithm for analysis of both respiratory and nonrespiratory related beat-to-beat changes in different ECG features.
\end{abstract}

Index Terms-ECG-derived respiration (EDR), principal component analysis (PCA).

\section{INTRODUCTION}

W E PRESENT an algorithm for analyzing beat-to-beat changes in features such as $\mathrm{P}$ waves, QRS complexes, and $\mathrm{T}$ waves from single-lead ECGs. The algorithm has potential use in identifying abnormal changes in these features, for example, $\mathrm{T}$ wave alternans, but here we seek to quantify the sensitivity of the algorithm to respiratory-induced variability of ECG features. We aim to show that the algorithm is able to track beat-to-beat changes in different ECG features and in doing so provides a surrogate respiratory signal comparable with other algorithms for ECG-derived respiration (EDR). EDR has been the focus of much research over the last 20 years [1]. This

Manuscript received September 29, 2008; revised January 14, 2009 and March 6, 2009. First published April 7, 2009; current version published March 24, 2010. This work was supported in part by the U.K. Engineering and Physical Sciences Research Council (EPSRC). The work of P. Langley was supported by the EPSRC Advanced Research Fellowship. Asterisk indicates corresponding author.

${ }^{*} \mathrm{P}$. Langley is with the Cardiovascular Physics and Engineering Research Group, Institute of Cellular Medicine, Newcastle University, Newcastle upon Tyne NE7 7DN, U.K., and also with the Freeman Hospital, Newcastle upon Tyne NE7 7DN, U.K. (e-mail: philip.langley@ncl.ac.uk).

E. J. Bowers is with the Medical Physics Department, Freeman Hospital, Newcastle upon Tyne NE7 7DN, U.K. (e-mail: emma.bowers@nuth.nhs.uk).

A. Murray is with the Medical Physics Department, Freeman Hospital, Newcastle upon Tyne NE7 7DN, U.K., and also with Newcastle University, Newcastle upon Tyne NE7 7DN, U.K. (e-mail: alan.murray@nuth.nhs.uk).

Digital Object Identifier 10.1109/TBME.2009.2018297 research is driven by the desire to reduce the number of sensors connected to patients during clinical studies, which require both respiratory and cardiac monitoring, for example, polysomnography [2]. The benefits include reduced complexity of instrumentation and increased patient comfort. Reducing the number of sensors is an important consideration in home and tele-based monitoring applications. Additionally, EDR allows the estimation of respiratory rate from archives of ECG databases where respiration was not recorded but subsequently has become an important parameter to measure. For example, baroreflex studies are highly sensitive to respiration rate but respiration has not been routinely recorded in many studies [3].

\section{A. Respiratory-Induced Modulation of ECG}

Respiratory-induced changes in the ECG arise due to several mechanisms. First, the electrical impedance of the thorax changes due to changes in lung volume [4]. Second, the heart vector changes due to changes in the displacement and orientation of the heart with respect to the ECG electrodes [5]. Third, heart rate changes due to respiratory-induced changes to the autonomic nervous system [6]. These factors give rise to morphological variation in ECG features related to the breathing cycle that we hope to capture in our algorithm.

\section{B. ECG-Derived Respiration Algorithms}

A number of algorithms for deriving respiration from the ECG have been described in the literature. These algorithms exploit the respiratory-induced changes of the ECG to provide a surrogate respiratory signal. By this we mean a signal with varying amplitude corresponding to the different phases of respiration [1]. From this, the respiratory rate and temporal pattern of breathing can be estimated. Many of these algorithms employ a multilead approach, which provide robust estimates of respiration, but can be implemented only where multilead ECG recordings are available [1]. The simplest single-lead EDR algorithms measure the beat-to-beat amplitude variation of the QRS complex [7], [8] or T wave [9] that are associated with the respiratory-induced variation in thoracic impedance [10]. However, such algorithms are susceptible to errors due to the inherent uncertainty in measuring amplitude when the ECG contains noise. A development to overcome the susceptibility of the amplitude-based algorithms to noise was the EDR algorithm based on the area under the QRS complex. This can be employed in single and multilead applications but requires accurate estimation of ECG baseline amplitude [11]. 
Respiratory-induced variations in heart rate provide another simple surrogate for respiration [1], but cannot be used in patients with abnormal heart rhythms or pacemakers.

\section{Principal Component Analysis}

Principal component analysis (PCA) is a technique that is generally used for reducing the dimensionality of multivariate datasets [12]. Considering a vector of $n$ random variables $\mathbf{x}$ for which the covariance matrix is $\boldsymbol{\Sigma}$, the principal components (PCs) can be defined by

$$
z=\mathbf{A} \boldsymbol{x}
$$

where $\boldsymbol{z}$ is the vector of $n$ PCs and $\mathbf{A}$ is the $n \times n$ orthogonal matrix with rows that are the eigenvectors of $\boldsymbol{\Sigma}$ [12]. The eigenvalues of $\boldsymbol{\Sigma}$ are proportional to the fraction of the total variance accounted for by the corresponding eigenvectors, so that the PCs explaining most of the variance in the original variables can be identified. If, as is usually the case, some of the original variables are correlated, a small subset of the PCs describes a large proportion of the variance of the original data. PCA has found widespread application in ECG signal processing [13]. These applications include noise estimation [14] and source separation applied to fetal ECG [15] and atrial fibrillation [16].

We hypothesize that beat-to-beat changes in ECG features such as QRS complex, T wave, or P wave could be identified by PCA from multibeat, single-lead ECG recordings. This hypothesis is based on the knowledge that beat-to-beat changes in ECG features result in a change in the correlation between these features at different beats. Since respiration is the main effect that modulates the ECG, our aim was to investigate whether PCA could detect respiratory-induced changes to ECG features and to assess PCA as a tool for deriving the respiratory signal from the single-lead ECG.

\section{METHODS}

\section{A. ECG and Respiratory Recordings}

1) Subjects and Data Acquisition: Twenty subjects with no known cardiac and respiratory diseases were recruited. The group comprised an equal number of male and female subjects. The mean age was 36 years with range of 21-60 years. ECG lead II and the respiratory signal were recorded from the subjects during controlled and natural breathing. The respiratory signal was obtained from magnetic displacement sensors placed on the back and chest of the subject that provided an indication of the respiratory cycle. ECG and the respiratory signal were digitally sampled at $500 \mathrm{~Hz}$ and stored on computer for subsequent analysis. During recordings, patients remained in the semisupine position. The data for one subject could not be analyzed because the high-amplitude QRS complexes exceeded the amplitude limit of the acquisition system causing clipping of this feature. One recording contained ectopic beats and was analyzed separately.

2) Breathing Patterns: All subjects were asked to breathe at six different rates: $4,6,8,10,12$, and 14 breaths per minute (bpm). Controlled breathing was facilitated by a display screen that displayed a scrolling triangular waveform at the specific breathing rate. The upward slope of the waveform indicated inspiration and the downward slope indicated exhalation. The subjects maintained each breathing pattern for $180 \mathrm{~s}$, and ECG and respiration sensor output were recorded during these intervals. An additional recording during natural breathing was obtained for each subject. All subjects followed the same breathing patterns but the order of the breathing patterns was randomized to avoid any bias arising from a training effect of the breathing sequence or fatigue.

\section{B. ECG-Derived Respiration}

Three algorithms for deriving the respiratory signal from the ECG were implemented: the PCA-based algorithm and two established algorithms, one based on RR intervals and one based on QRS amplitude. The established algorithms, alongside the direct respiratory measurements, provided an objective assessment of the accuracy of the respiratory signals obtained from the PCA algorithm and facilitated comparisons of this algorithm's performance against the other algorithms.

1) Preprocessing of ECG: Each algorithm required the ECG beats to be detected. An approximate time marker for each beat was obtained as the maximum rate of change of the ECG by identifying maxima in the differential of the ECG signal. A search around these time markers for maximum values in the ECG provided accurate measurement of $\mathrm{R}$ wave time points

$$
r(i), \quad i=1,2, \ldots, n
$$

where $n$ is the number of beats. To validate the detected $\mathrm{R}$ waves, the ECGs were plotted, and accurate detection was confirmed in all cases by visual inspection. Additionally, ectopic beats were identified from these plots, and the ECGs containing ectopic beats were analyzed separately from those without.

2) ECG-Derived Respiration From RR Intervals and $Q R S$ Amplitude: The two established algorithms derived the respiratory signal from: 1) RR interval and 2) QRS amplitude. For the RR interval algorithm, an RR interval time series was generated for each ECG as

$$
r r(i)=r(i+1)-r(i), \quad i=1,2, \ldots, n-1 .
$$

For the QRS amplitude algorithm, the amplitude was measured as the amplitude difference between the foot of the $S$ wave and peak of the $\mathrm{R}$ wave

$$
\operatorname{amp}(i)=r_{\mathrm{amp}}(i)-s_{\mathrm{amp}}(i), \quad i=1,2, \ldots, n
$$

where $r_{\mathrm{amp}}$ and $s_{\mathrm{amp}}$ are the amplitudes of the R and $\mathrm{S}$ waves, respectively. $s_{\text {amp }}$ was measured as the minimum amplitude in a window of 40 sample points $(80 \mathrm{~ms})$ after the $\mathrm{R}$ wave peak. EDR derived from the RS amplitude has been shown to be more robust than measuring the amplitude with respect to the baseline [8].

3) ECG Respiration From Principal Component Analysis: PCA is applied to multivariate datasets. In our application, the multivariate dataset is the aligned collection of beat features from single-lead ECG recordings. The algorithm can be applied to any ECG feature, i.e., $\mathrm{P}$ wave, QRS complex, T wave, or 


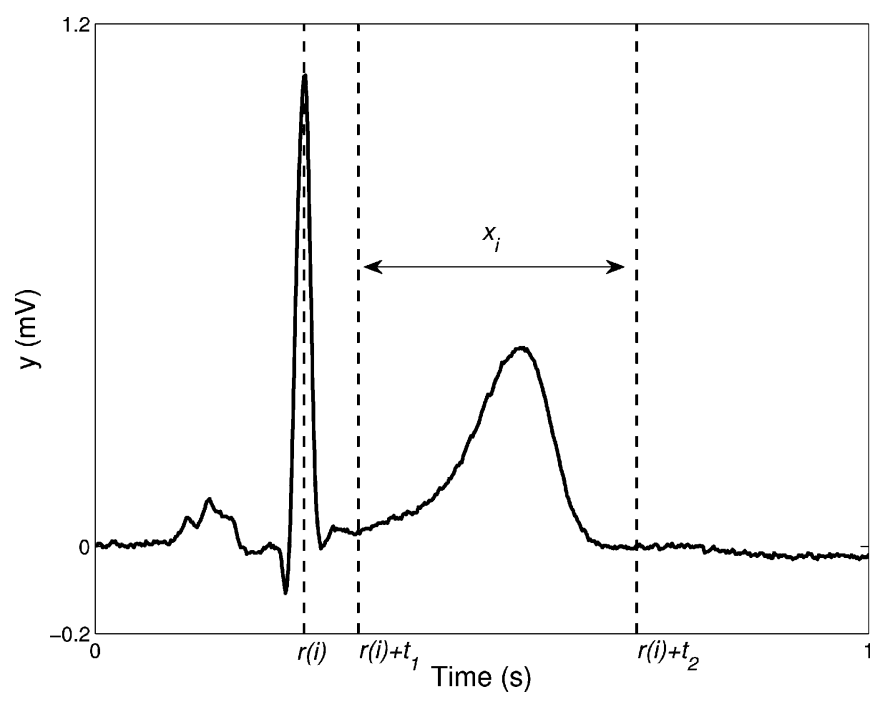

Fig. 1. Identification of ECG feature $x_{i}$ : in this example, the $\mathrm{T}$ wave at beat $i$. The feature window is defined by $t_{1}$ and $t_{2}$, which are the offsets relative to the time point of the $\mathrm{R}$ wave peak indicated by $r(i)$.

the full ECG cycle, so we evaluated the algorithm on all these features separately.

a) Identifying beat features: The ECG segments containing the specific feature were identified automatically using a fixed window relative to the detected $\mathrm{R}$ waves

$$
x_{i}(t)=y(t) \quad r(i)+t_{1}<t<r(i)+t_{2}, i=1,2, \ldots, n
$$

where $x_{i}$ is the feature at beat $i, y$ is the ECG, and $t_{1}$ and $t_{2}$ are the time points that define the length of the feature window. An example is illustrated in Fig. 1. The algorithm did not require accurate detection of start and end points of the features, so $t_{1}$ and $t_{2}$ were fixed for each feature across all beats and across all subjects.

b) Applying PCA: The collection

$$
\boldsymbol{X}(t)=\left[x_{1}(t), x_{2}(t), x_{3}(t), \ldots x_{n}(t)\right]
$$

is the time-ordered collection of the feature at all beats into a single matrix to which PCA can be applied. The means of the $x_{i}$ are removed and the covariance matrix computed.

Defining the covariance matrix

$$
\Sigma=\operatorname{cov}(\boldsymbol{X})
$$

the solution to

$$
\Sigma \boldsymbol{\alpha}_{j}=\lambda_{j} \boldsymbol{\alpha}_{j}, \quad j=1,2, \ldots, n
$$

yields the eigenvectors $\left(\boldsymbol{\alpha}_{j}\right)$ and eigenvalues $\left(\lambda_{j}\right)$, which were computed using numerical analysis software (MATLAB).

The PCs were obtained using

$$
z_{j}=\boldsymbol{\alpha}_{j} \boldsymbol{x}, \quad j=1,2, \ldots, n
$$

and arranged in order of magnitude of eigenvalue. The PCs are a linear transformation of the beats with transformation coefficients given by the eigenvectors $\boldsymbol{\alpha}_{j}$. It is the eigenvectors that provide the surrogate respiratory signal in our analysis.

In addition to establishing which particular feature of the ECG provided the best respiratory signal for the PC-based algorithm, we also sought to establish which PCs yielded the eigenvectors most sensitive to respiratory changes. PCA provides as many PCs as there are analyzed beats (for example, approximately 180 in a 180-s recording); however, because these beats are highly correlated and the respiratory-related ECG changes are large, most of the variability was expressed by the first few PCs. Therefore, we assessed the eigenvectors of the first three PCs as surrogates for the respiratory signal for all ECG features.

\section{Comparisons of ECG-Derived Respiration With Respiratory Signal}

For each subject recording, we computed 14 EDR signals. These comprised one from the RR algorithm, one from the QRS amplitude algorithm, and 12 from the PCA algorithm, which was applied separately to four ECG features (whole beat, P wave, QRS complex, and T wave), and the eigenvectors from the first three PCs were analyzed for each feature. These were labeled RR, Amp, $\mathrm{PC}_{j}^{\mathrm{WB}}, \mathrm{PC}_{j}^{\mathrm{P}}, \mathrm{PC}_{j}^{\mathrm{QRS}}$, and $\mathrm{PC}_{j}^{\mathrm{T}}(j=1,2,3)$, respectively. So, for 19 subjects, each recorded at seven breathing rates, we analyzed a total of 1862 EDR signals. Before comparing the EDR with the respiratory signal, the beat-wise samples of the EDR were resampled to the same sample rate as for the respiratory signal $(500 \mathrm{~Hz})$ using linear interpolation. Similarity in the time domain between EDR and the respiratory signal was quantified using cross-correlation. The maximum absolute correlation was determined for each recording; hence, correlation was unaffected by differences in phase between the ECG-derived signal and the respiratory signal. The frequencydomain measure of similarity, the magnitude squared coherence, was also estimated for each recording

$$
C_{x y}(f)=\frac{\left|P_{x y}(f)\right|^{2}}{P_{x}(f) P_{y}(f)}
$$

where $P_{x}$ and $P_{y}$ are the power spectral densities of the respiratory and surrogate respiratory signals and $P_{x y}$ is the cross power spectral density. We determined the maximum coherence that occurred at the respiratory frequency [17]. The spectra were calculated using Welch's method using a $2^{14}$ point fast Fourier transform (FFT) with a periodic Hamming window. The length of the window was chosen to obtain eight equal sections of input signal, and $50 \%$ overlap was used for computing the spectra.

\section{Statistical Analysis}

The algorithms (RR, Amp, and 12 variations of the PCA algorithm) were ranked according to the median values of coherence and correlation across subjects and breathing rates. Tests for significant differences in correlation and coherence between the highest ranking implementation of the PCA algorithm with those for the RR and Amp algorithms were conducted using the Kruskal-Wallis test, which was compensated for multiple comparisons by the Tukey's honestly significant difference criterion. 
ECG
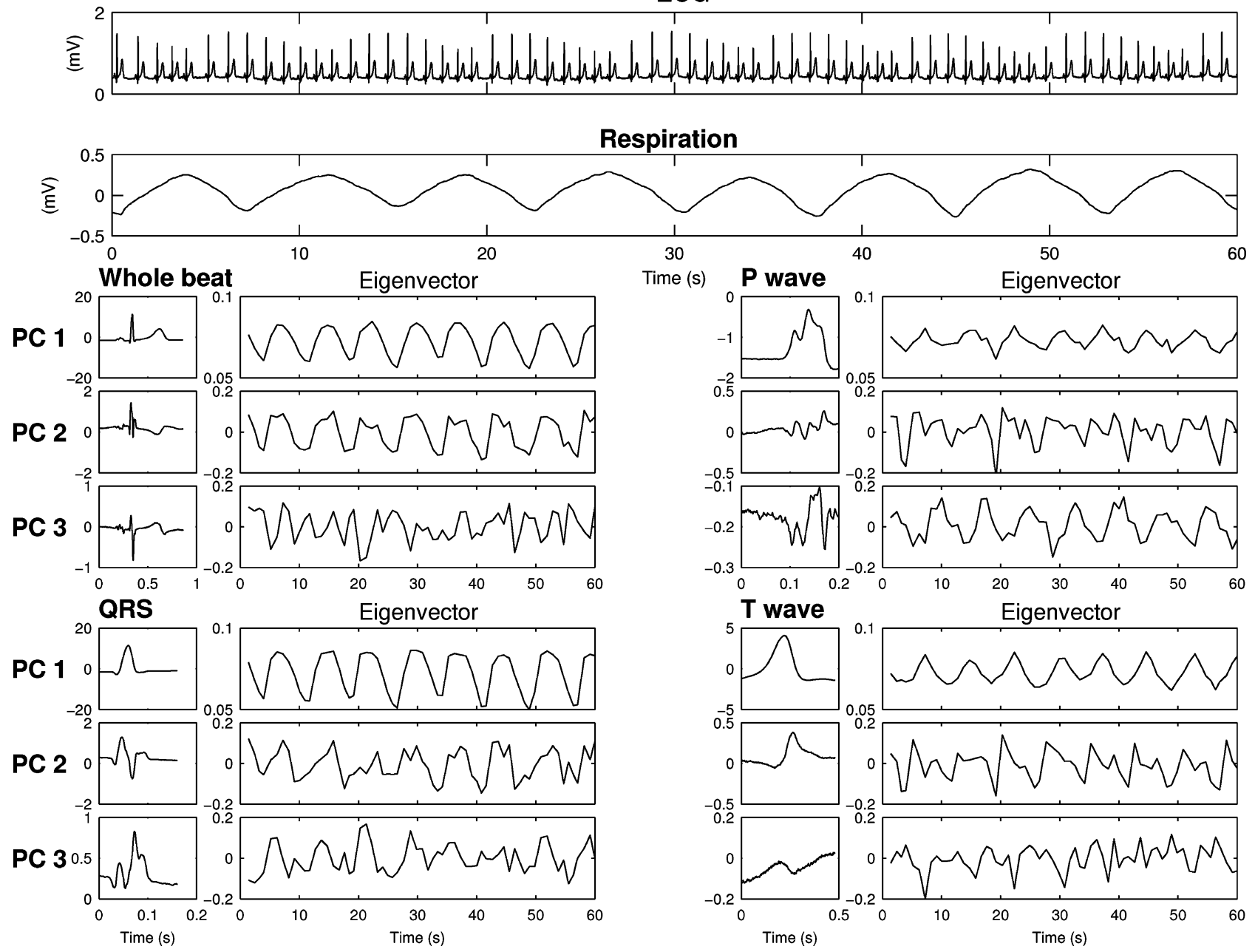

Fig. 2. ECG and respiratory sensor signal from a subject breathing at $8 \mathrm{bpm}$ along with the first three PCs and their eigenvectors for each of the ECG features. In the top panel, the ECG shows clear amplitude modulation corresponding to the respiratory signal obtained from the output of the magnetic respiratory sensor shown in the second panel. In the remaining panels, the first three PCs and their corresponding eigenvectors obtained from the PCA algorithm when applied to the whole beat, $\mathrm{P}$ wave, QRS complex, and T wave are shown. The eigenvector of the first PC clearly reflects the respiratory pattern for all ECG features.

\section{RESULTS}

\section{A. EDR From PCA}

Fig. 2 provides an example of the first three PCs and their eigenvectors obtained from different features of the ECG for a subject breathing at $8 \mathrm{bpm}$. Note that the first PC, which is approximately ten times greater than the other PCs, represents the "typical" beat morphology for the particular feature across all the beats. The values of the eigenvector of the first PC are all positive with cyclic variation corresponding to the respiratory signal measured from the subject. The second and third PCs describe subtle morphological differences not described by the first PC, and some of their eigenvectors also exhibit cyclic variations corresponding to the respiratory rate. The eigenvectors can be seen to vary at a rate corresponding to the respiratory signal for at least one of the PCs for each feature.

\section{B. Comparison of Algorithms}

Fig. 3 compares the performance of each algorithm in terms of coherence and correlation for each breathing pattern. The best feature for the PCA algorithm was the respiratory estimate from the first $\mathrm{PC}$ of the $\mathrm{QRS}$ complex $\left(\mathrm{PC}_{1}^{\mathrm{QRS}}\right)$. This was closely followed by the respiratory estimates from the first $\mathrm{PC}$ of the whole beat $\left(\mathrm{PC}_{1}^{\mathrm{WB}}\right)$, first $\mathrm{PC}$ of the $\mathrm{T}$ wave $\left(\mathrm{PC}_{1}^{\mathrm{T}}\right)$, and the third $\mathrm{PC}$ of the $\mathrm{QRS}$ complex $\left(\mathrm{PC}_{3}^{\mathrm{QRS}}\right)$. These implementations of the PCA algorithm gave results that were either better or the same as the RR or amplitude-based algorithms, as illustrated in Table I. The coherence and correlation values for the PCA algorithm $\left(\mathrm{PC}_{1}^{\mathrm{QRS}}\right)$ were significantly greater than the $\mathrm{RR}$ algorithm ( $p<0.05$ and $p<0.0001$, respectively), but these were not significantly different to values for the amplitude algorithm. There were no significant differences for these algorithms between the coherence and correlation values obtained for the different breathing patterns.

For most of the recordings, the ECGs showed significant respiratory-induced QRS amplitude changes. Fig. 4 shows an example in which the respiratory-induced QRS amplitude changes were small. This figure shows the ECG, chest sensor signal, and EDR for RR, amplitude, and eigenvectors of the first and third PCs of QRS complexes. Clearly, neither the amplitude 
Rate

Coherence

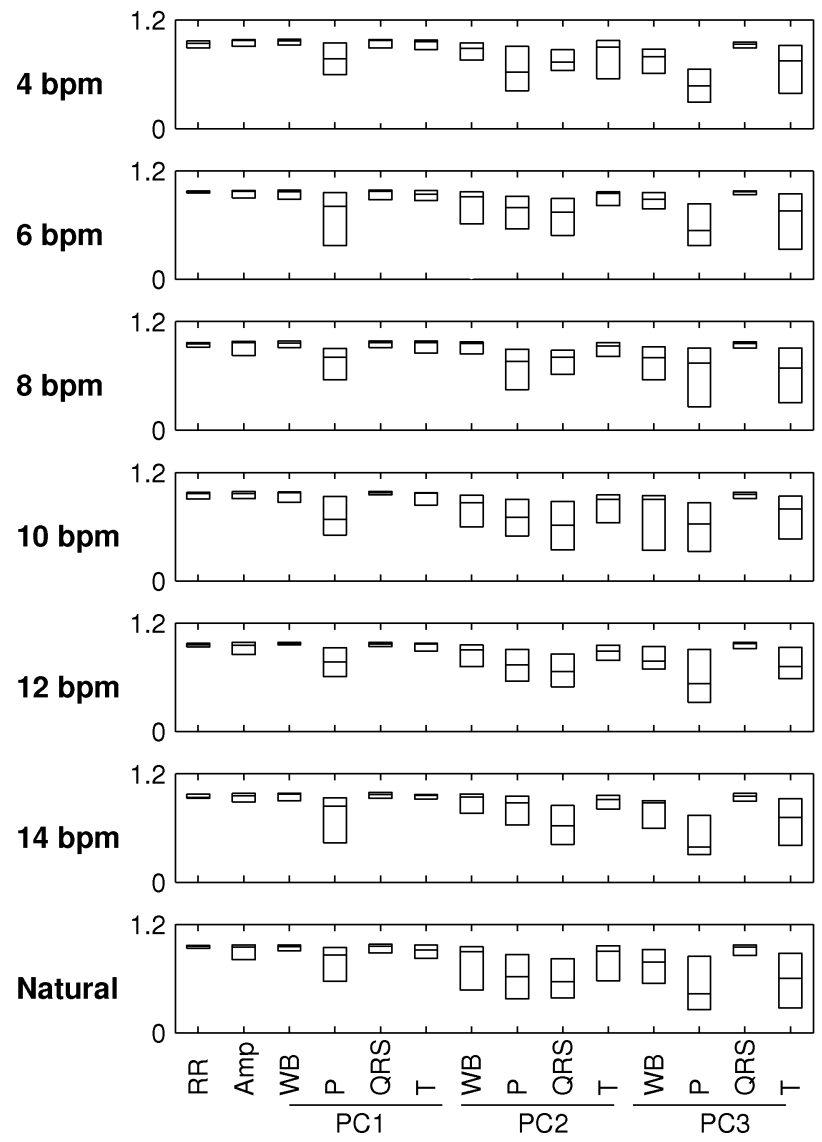

Correlation
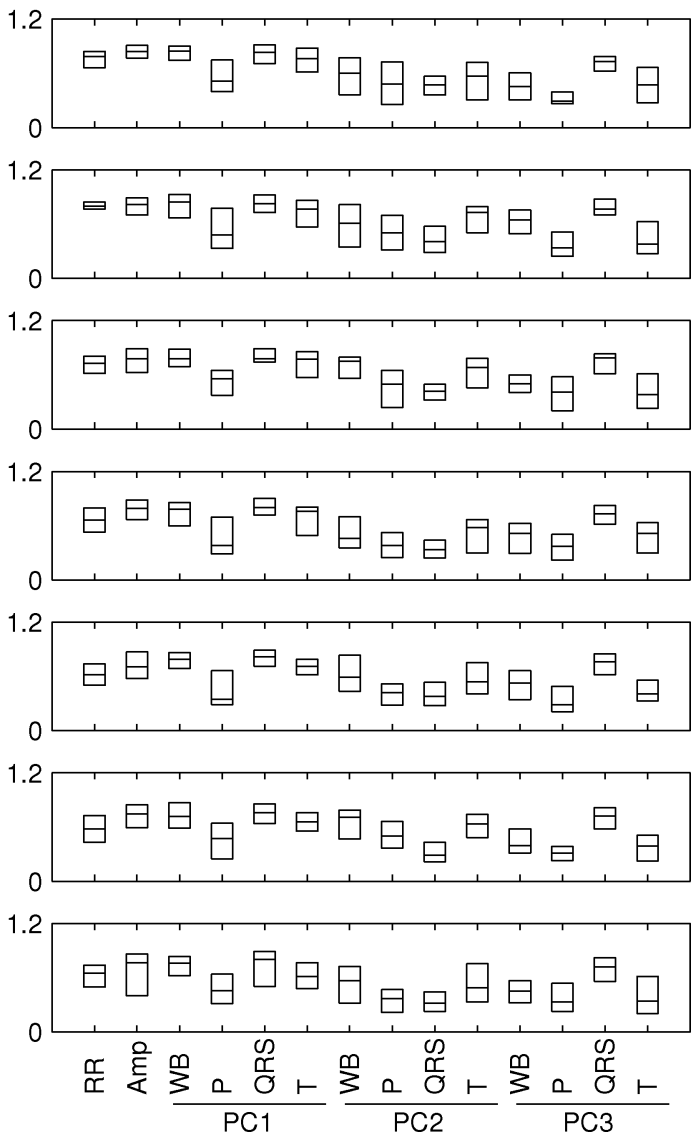

Fig. 3. Coherence and correlation of the EDR to the respiratory signal for each algorithm and breathing rate (bpm). The label "Natural" indicates subjects breathing naturally. Box and line indicate median and interquartile range, respectively. WB: whole beat, RR: RR-based algorithm, Amp: QRS-amplitude-based algorithm.

TABLE I

TOP SiX RANKING ALgORITHMS FOR COHERENCE AND CORRELATION

\begin{tabular}{lllll}
\hline & \multicolumn{2}{c}{ Coherence } & \multicolumn{2}{c}{ Correlation } \\
\hline Rank & Algorithm & Value & Algorithm & Value \\
\hline 1 & $\mathrm{PC}_{1}{ }^{\mathrm{QRS}}$ & 0.97 & $\mathrm{PC}_{1}{ }^{\mathrm{QRS}}$ & 0.80 \\
2 & $\mathrm{PC}_{1}{ }^{\mathrm{WB}}$ & 0.97 & $\mathrm{PC}_{1}{ }^{\mathrm{WB}}$ & 0.78 \\
3 & $\mathrm{Amp}$ & 0.96 & $\mathrm{Amp}$ & 0.78 \\
4 & $\mathrm{PC}_{1}{ }^{\mathrm{T}}$ & 0.96 & $\mathrm{PC}_{1}{ }^{\mathrm{T}}$ & 0.76 \\
5 & $\mathrm{RR}$ & 0.96 & $\mathrm{PC}_{3}{ }^{\mathrm{QRS}}$ & 0.73 \\
6 & $\mathrm{PC}_{3}{ }^{\text {}}{ }^{\mathrm{RS}}$ & 0.96 & $\mathrm{RR}$ & 0.66 \\
\hline
\end{tabular}

algorithm nor the eigenvector of the first PC provided good estimates of the respiratory signal with correlations of 0.56 and 0.58 and coherences of 0.77 and 0.89 , respectively. However, the eigenvector of the third PC of QRS complexes achieves a correlation of 0.78 and coherence of 0.99 that outperformed all the other algorithms.

\section{Effect of Atrial Ectopic Beats}

There were no ventricular ectopic beats, but one of the recordings contained atrial ectopic beats and this was analyzed separately to assess the sensitivity of the PCA algorithm to such beats. The morphology of the ventricular complex of the ectopic beats was similar to that of the regular beats. Fig. 5 compares the respiratory signals obtained from the algorithms from this recording. The artifact introduced into the respiratory signal derived from the PCA algorithm is much smaller than the respiratory variation; hence, the PCA algorithm was robust against these ectopic beats. As would be expected, the RR-based algorithm is not a robust surrogate for the respiratory signal in the presence of ectopic beats, and further processing would be required to remove the effect of the abnormal beat intervals. Since the morphology of the QRS complex of the atrial ectopic beats was similar to that of the regular beats, the EDR derived from the amplitude algorithm showed only slight disturbance due to the ectopic beats. As is the case for all other EDR algorithms, where the ectopic beat morphology is substantially different from the normal beat morphology, for example, premature ventricular beats, it would be beneficial to remove these beats before applying the PCA algorithm and to use interpolation to estimate the missing data in the surrogate respiratory signal.

\section{Reconstruction of Beats From Principal Components to Investigate Respiration-Induced Morphology Changes}

We investigated the respiratory-induced morphology changes to ECG beats by reconstructing two beats, one corresponding 

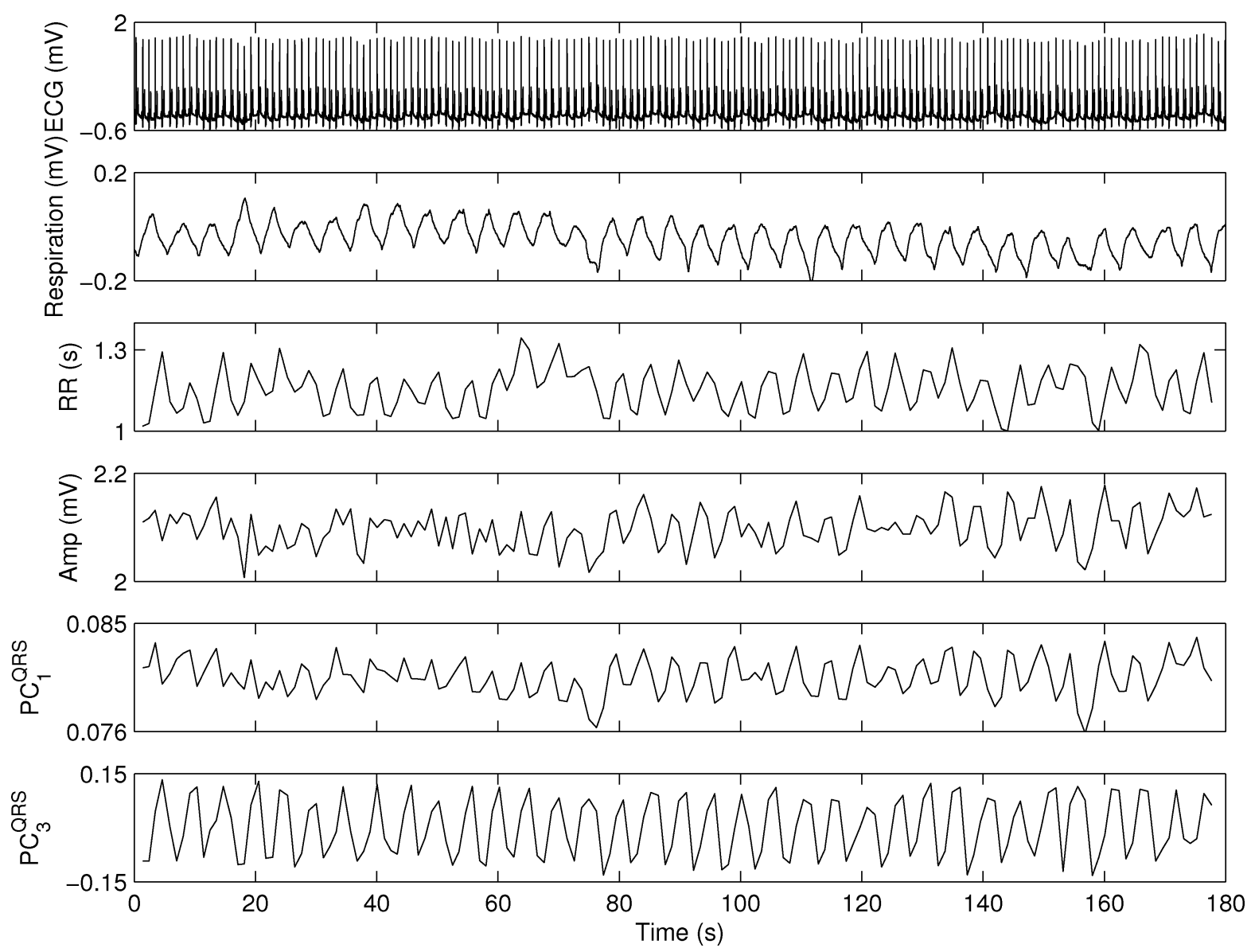

Fig. 4. Respiratory surrogates derived from the algorithms in an ECG with relatively small respiratory-induced QRS amplitude changes. The ECG is from a subject breathing at $12 \mathrm{bpm}$ and the amplitude changes were less than $0.2 \mathrm{mV}$. The output from the respiratory sensor is shown along with surrogate respiratory

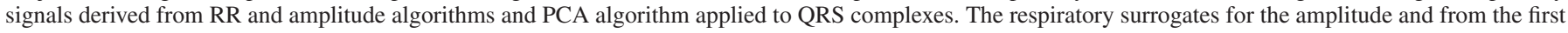
$\mathrm{PC}$ are highly correlated and are not good estimates of the respiratory signal. The third PC provides a better estimate.

to the peaks of the respiratory cycle and one corresponding to the troughs of the respiratory cycle. Using the example shown in Fig. 2 for the PCA algorithm applied to the whole beat, we first identified the PCs with eigenvectors that showed the clearest respiratory-related changes. Using these PCs only, we performed an inverse transform using transform coefficients obtained from the peaks and troughs of the eigenvectors to recreate beats corresponding to different phases of the respiratory cycle. The eigenvectors of the first two PCs showed clear respiratory-related changes and were used in the inverse transformation. Performing the inverse transformation with these PCs isolated the effect of respiratory-induced ECG changes from other factors not related to respiration, which were described by the remaining PCs. The peaks and troughs of the eigenvector of the first PC were $0.09\left(c_{1 \text {,peak }}\right)$ and $0.06\left(c_{1, \text { trough }}\right)$, respectively. The corresponding values in the eigenvector of the second PC were $0.1\left(c_{2, \text { peak }}\right)$ and $-0.1\left(c_{2, \text { trough }}\right)$. Using these coefficients, we reconstructed the beats $x_{\text {peak }}$ and $x_{\text {trough }}$, which are approximations of the beat morphology at different phases of the respiratory cycle

$$
\begin{gathered}
x_{\text {peak }}=z_{1} c_{1, \text { peak }}+z_{2} c_{2, \text { peak }} \\
x_{\text {trough }}=z_{1} c_{1, \text { trough }}+z_{2} c_{2, \text { trough }} .
\end{gathered}
$$
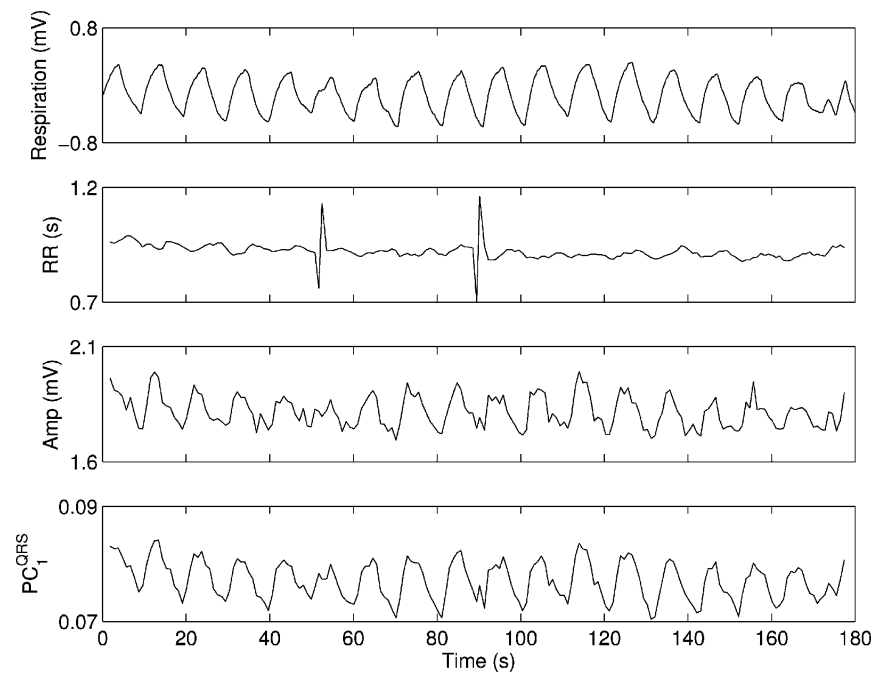

Fig. 5. Respiration sensor output signal and EDR from a subject breathing at $6 \mathrm{bpm}$ for $180 \mathrm{~s}$. There were two atrial ectopic beats as indicated by the spikes in the RR-derived respiratory signal. The respiratory estimate from the first PC of QRS complexes was unaffected by the atrial ectopic beats.

The reconstructed beats are illustrated in Fig. 6. The reconstructed beats clearly show the large amplitude changes that occurred due to respiration and also the more subtle changes 


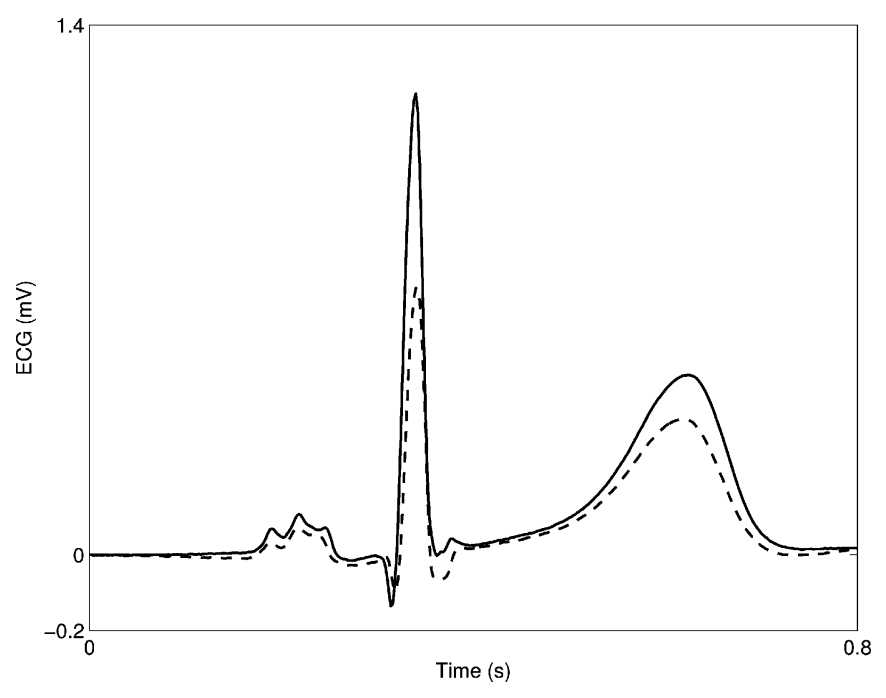

Fig. 6. Reconstruction of ECG beats to illustrate the beat morphology at different phases of the respiratory cycle using the inverse PCA transformation. Solid line is the ECG beat reconstructed using coefficients corresponding to peaks in the respiratory cycle and the dashed line is the ECG beat reconstructed using coefficients at the troughs of the respiratory cycle. The beats were reconstructed using only $\mathrm{PCs}$ whose eigenvectors showed clear respiratory variation $\left(\mathrm{PC}_{1}^{\mathrm{W}} \mathrm{B}\right.$ and $\mathrm{PC}_{2}^{\mathrm{WB}}$ ), and the coefficients for the inverse transform were obtained from eigenvector values corresponding to the peaks and troughs of the respiratory cycle.

to beat morphology, for example, changes in QRS duration and timing of the peak of the $\mathrm{T}$ wave. The amplitude changes were described by the first PC and its eigenvector and the subtle morphological changes were described by the second PC and eigenvector. Note that the eigenvector of the first PC was always positive and the eigenvector of the second PC contained both positive and negative values. Hence, the eigenvector of the first PC always described amplitude changes and the eigenvector of the second PC described the subtle morphological changes in the ECG. In this regard, the PCA algorithm can be considered to take account of and separate both the amplitude and morphological changes associated with respiration.

\section{E. Nonrespiratory-Related ECG Changes}

Our analysis revealed other potential applications of the algorithm for analysis of beat-to-beat changes in the ECG other than those due to respiration. Fig. 7 illustrates the eigenvectors from the PCA algorithm applied to $\mathrm{P}$ waves from a subject breathing at $14 \mathrm{bpm}$. The eigenvectors of the second PC exhibited clear respiratory variations. The eigenvectors of the first PC exhibited large variations unrelated to the respiratory signal. These were found to reflect large variations in $\mathrm{P}$ wave amplitudes that occurred throughout the recording, as illustrated in Fig. 7. There were no corresponding amplitude changes in the other ECG features and the cause of these $\mathrm{P}$ wave amplitude variations was unknown in this subject, but similar observations of spontaneous changes in $\mathrm{P}$ waves have been reported previously [18]. This demonstrates the utility of the PCA algorithm for simultaneously tracking respiratory and nonrespiratory beat-to-beat variations in ECG beat features.

\section{DISCUSSION}

PCA is a novel approach to EDR. We have demonstrated its application using the different features of the ECG and obtained significantly better results than the RR-based algorithm. The PCA algorithm gave the best surrogate to the respiratory signal when applied to QRS complexes, and good results were also obtained when applied to other ECG features such as T waves. The eigenvector of the first PC was sensitive to respiratory changes, and in most recordings, these were associated with amplitude changes. However, unlike other algorithms, the PCA algorithm also extracts the subtle morphological changes to ECG features, which were expressed in the second and third PCs. The PCA-based algorithm also has the advantage that dominant PCs are relatively noise free because ECG noise is uncorrelated to the ECG features and is separated into the lower PCs. This is demonstrated by the reconstructed beats shown in Fig. 6 where the first two PCs were used to reconstruct the beats at the different phases of the respiratory cycle.

There was no significant difference between the PCA algorithm $\left(\mathrm{PC}_{1}^{\mathrm{QRS}}\right)$ and the amplitude algorithm, which could be expected since the eigenvector of the first PC is closely associated with the amplitude of the QRS complex. However, an interesting finding was that morphological changes in QRS complexes expressed by the third PC were also highly correlated to respiration, indicating that the algorithm captures the subtle morphological beat-to-beat differences as well as the large amplitude changes. It was shown that the algorithm was successful at identifying small changes in ECG features even if there were no large amplitude variations related to respiration. Although in general the algorithm when applied to $\mathrm{P}$ waves did not give results as good as the other features or algorithms, there were examples where the $\mathrm{P}$ wave gave results better than the existing algorithms and suggests that the algorithm could be applied to investigate respiratory-related changes to the $\mathrm{P}$ wave that have not been investigated previously. More interestingly, in one subject, in addition to providing a good respiratory surrogate from the $\mathrm{P}$ wave, the algorithm detected changes in $\mathrm{P}$ wave morphology not related to respiration. This demonstrates the capability of the algorithm to separate the respiratory and nonrespiratory changes in beat morphology and demonstrates the wider application of this algorithm as a general tool for analysis of beat-to-beat changes in ECG features. Such a tool might be useful in serial ECG analysis. We have already demonstrated the accuracy of the algorithm applied to the detection of T wave alternans [19].

Here, our aim was to assess the potential of the new algorithm for providing a surrogate for the respiratory signal in controlled conditions and to evaluate it against some existing algorithms. As such, we have restricted our analysis to healthy individuals and further work will test the algorithm in clinical practice. Future work should assess the sensitivity of the algorithm to different leads as only a single-lead position was used in our analysis and choice of lead is an important consideration [11]. In our analysis, we identified the PCs yielding the respiratory surrogate by comparison with the known respiratory signal from the magnetic chest sensors. In general, the 

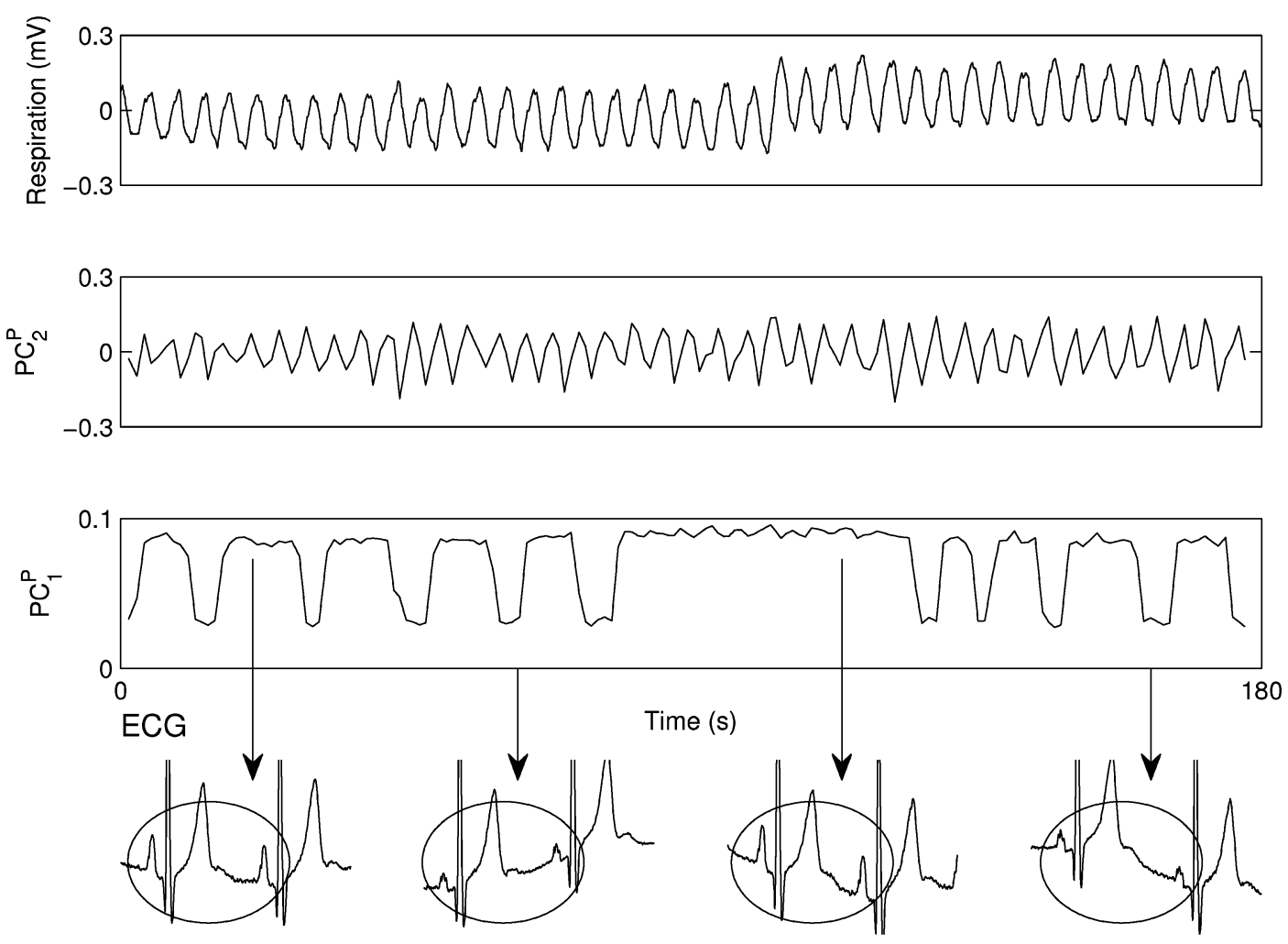

Fig. 7. Respiratory sensor signal and PC eigenvectors obtained from the algorithm applied to $\mathrm{P}$ waves in a subject breathing at $14 \mathrm{bpm}$ for $180 \mathrm{~s}$. The eigenvector of the second PC gave a good representation of the respiratory signal (coherence $=0.98$, correlation $=0.77$ ). The eigenvector of the first PC showed large variations unrelated to respiration. These are due to changes in P wave amplitude as illustrated by the ECG segments beneath. The ECG segments are $2 \mathrm{~s}$ extracts from the continuous ECG shown on a 1-mV peak-to-peak scale. Segments are shown that correspond to high and low values of the eigenvector of the first PC. The large variation in $\mathrm{P}$ wave amplitude can be seen in the different segments. The $\mathrm{P}$ wave amplitude changes were unrelated to respiration.

eigenvector of the first PC yielded the best surrogate. In the intended application, without a respiratory sensor, the eigenvector of the first PC would be a good candidate for the respiratory surrogate. Fortunately, respiratory-induced changes in ECG features tend to be cyclic (at the respiratory rate), and respiratory rate tends to be fairly constant even during natural breathing. In contrast, nonrespiratory-induced changes in ECG features are often sporadic, or if regular, outside the physiological rate for respiration (e.g., $\mathrm{T}$ wave alternans). This facilitates the identification of components containing respiratory- and nonrespiratory-induced changes. Our algorithm was robust against atrial ectopic beats where the beat morphology was similar to the regular beats but further testing would be needed for ectopics with different morphologies. It would have been impractical to evaluate our algorithm against all existing EDR algorithms, so we chose two well-defined, and hence, easily reproducible algorithms to test against. These are simple algorithms and further testing against more sophisticated algorithms should be undertaken. The clinical utility of our algorithm needs to be investigated but other EDR algorithms have found application in investigations of sleep disorders where the number of sensors attached to the patient is an important consideration [9]. Notwithstanding these limitations, our study demonstrates PCA as a novel algorithm for assessing both respiratoryand nonrespiratory-related beat-to-beat changes in different features of the ECG.

\section{REFERENCES}

[1] R. Bailón, L. Sörnmo, and P Laguna, ECG-derived respiratory frequency estimation in Advanced Methods and Tools for ECG Data Analysis, G. D. Clifford, F. Azuaje, and P. E. McSharry, Eds. London, U.K.: Artech House, 2006, pp. 215-243.

[2] C. A. Kushida, M. R. Littner, T. Morgenthaler, C. A. Alessi, D. Bailey, J. Coleman, L. Friedman, M. Hirshkowitz, S. Kapen, M. Kramer, T. LeeChiong, D. L. Loube, J. Owens, J. P. Pancer, and M. Wise, "Practice parameters for the indications for polysomnography and related procedures: An update for 2005," Sleep, vol. 28, no. 4, pp. 499-521, Apr. 2005

[3] L. Bernardi, C. Porta, L. Spicuzza, J. Bellwon, G. Spadacini, A. W. Frey, L. Y. C. Yeung, J. E. Sanderson, R. Pedretti, and R. Tramarin, "Slow Breathing increases arterial baroreflex sensitivity in patients with chronic heart failure," Circulation, vol. 105, pp. 143-145, 2002.

[4] G. Hahn, I. Šipinková, F. Baisch, and G. Hellige, "Changes in the thoracic impedance distribution under different ventilatory conditions," Physiol. Meas., vol. 16, no. 3A, pp. A161-A173, Aug. 1995.

[5] R. Pallas-Areny, J. Colominas-Balague, and F. J. Rosell, "The effect of respiration-induced heart movements on the ECG," IEEE Trans. Biomed. Eng., vol. 36, no. 6, pp. 585-590, Jun. 1989.

[6] D. L. Eckberg, "The human respiratory gate," J. Physiol., vol. 548, no. 2, pp. 339-352, Apr. 2003.

[7] D. Dobrev and I. Daskalov, "Two-electrode telemetric instrument for infant heart rate and apnea monitoring," Med. Eng. Phys., vol. 20, no. 10, pp. 729-734, Dec. 1998.

[8] C. L. Mason and L. Tarassenko, "Quantitative assessment of respiratory derivation algorithms," in Proc. 23rd Annu. IEEE EMBS Int. Conf., 2001, pp. 1998-2001.

[9] B. Raymond, R. M. Cayton, R. A. Bates, and M. Chappell, "Screening for obstructive sleep apnoea based on the electrocardiogram-The computers in cardiology challenge," Comput. Cardiol., vol. 27, pp. 267-270, 2000. 
[10] H. Engblom, J. Foster, T. Martin, B. Groenning, O. Pahlm, H. Dargie, G. Wagner, and H. Arheden, "The relationship between electrical axis by 12-lead electrocardiogram and anatomical axis of the heart by cardiac magnetic resonance in healthy subjects," Amer. Heart J., vol. 150, pp. 507$512,2005$.

[11] G. B. Moody, R. G. Mark, A. Zoccola, and S. Mantero, "Derivation of respiratory signals from multi-lead ECGs," Comput. Cardiol., vol. 12, pp. 113-116, 1985.

[12] I. T. Jolliffe, Principal Component Analysis. New York: SpringerVerlag, 1986.

[13] F. Castells, P. Laguna, L. Sörnmo, A. Bollmann, and J. M. Roig, "Principal component analysis in ECG signal processing," EURASIP J. Adv. Signal Process., vol. 2007, p. 98, 2007.

[14] G. B. Moody and R. G. Mark, "QRS morphology representation and noise estimation using the Karhunen-Loeve transform," Comput. Cardiol., vol. 16, pp. 269-272, 1989.

[15] P. P. Kanjilal, S. Palit, and G. Saha, "Fetal ECG extraction from singlechannel maternal ECG using singular value decomposition," IEEE Trans. Biomed. Eng., vol. 44, no. 1, pp. 51-59, Jan. 1997.

[16] P. Langley, J. J. Rieta, M. Stridh, J. Millet-Roig, L. Sörnmo, and A. Murray, "Comparison of atrial signal extraction algorithms in 12-lead ECGs with atrial fibrillation," IEEE Trans. Biomed. Eng., vol. 53, no. 2, pp. 343-346, Feb. 2006.

[17] L. Sörnmo and P. Laguna, Bioelectrical Signal Processing in Cardiac and Neurological Applications. London, U.K./New York: Elsevier/Academic, 2005, pp. 614-618, ch. 8.

[18] D. A. Brody, M. D. Woolsey, and R. C. Arzbaecher, "Application of computer techniques to the detection and analysis of spontaneous P-wave variations," Circulation, vol. 36, pp. 359-371, 1967.

[19] D. Zheng, S. Stevens, P. Langley, K. Wang, A. J. Haigh, and A. Murray, "T-wave alternans: A comparison of different measurement techniques," Comput. Cardiol., vol. 35, pp. 597-600, 2008.

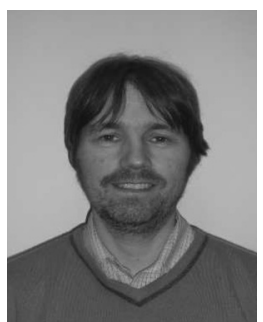

Philip Langley received the B.Eng. and Ph.D. degrees in electronic engineering from the University of Hull, Hull, U.K., in 1991 and 1996, respectively.

In 1998, he joined the Cardiovascular Physics and Engineering Research Group, Medical Physics Department, Freeman Hospital, Newcastle upon Tyne, U.K., where he is currently a Principal Research Associate and an Engineering and Physical Sciences Research Council (EPSRC) Advanced Research Fellow. He is also with the Institute of Cellular Medicine, Newcastle University, Newcastle upon Tyne, where he is engaged in research on cardiovascular electrophysiology and biomedical signal processing.

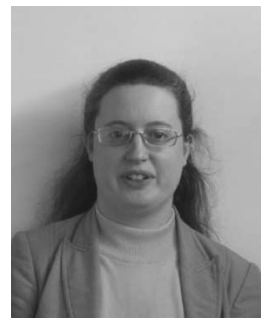

Emma J. Bowers received the M.Eng. degree in electronic systems engineering from the University of York, York, U.K., in 1996, the M.Sc. degree in medical physics from Surrey University, Surrey, U.K., in 1997, and the Ph.D. degree from Newcastle University, Tyne and Wear, U.K., in 2008.

She is engaged in research on the interaction between breathing, RR intervals, and systolic pressure changes. Since 2000, she has been a member of the Cardiovascular Physics and Engineering Research Group, Regional Medical Physics Department, Freeman Hospital, Newcastle upon Tyne, U.K. She has most recently been funded by the Institute of Physics and Engineering through a research training fellowship.

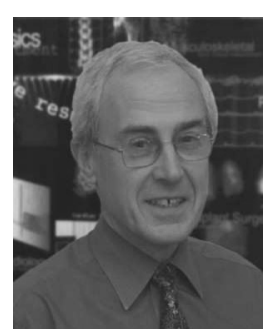

Alan Murray received the B.Sc. degree in electrical engineering from the University of Strathclyde, Glasgow, U.K., in 1968, and the Ph.D. degree in medical physics from the University of Edinburgh, U.K., in 1971.

$\mathrm{He}$ is currently the Clinical Director of Regional Medical Physics Department, Freeman Hospital, Newcastle upon Tyne, U.K. He is also a Professor of cardiovascular physics at Newcastle University, Newcastle upon Tyne. 